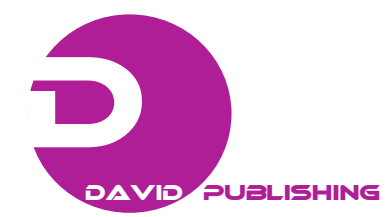

\title{
Growth, Inactivation and Toxin Production of Bacillus cereus in Cooked Rice under Different Temperatures
}

Jun Wang*, Seung-Ya Yang, Hyeon-Kyung Ham, Myoung-Su Park, Xi-Hong Zhao, Joong-Hyun Park, Fereidoun Forghani, Gwang-Hee Kim, Bo-Geum Park and Deog-Hwan Oh

Department of Food Science and Biotechnology and Institute of Bioscience and Biotechnology, Kangwon National University,

Chuncheon, Gangwon 200-701, Republic of Korea

*Corresponding author's e-mail: deoghwa@kangwon.ac.kr

\begin{abstract}
Bacillus cereusis capable of producing enterotoxin and emetic toxin that cause severe nausea, vomiting and diarrhea. The objective of this study was to investigate the effect of temperature on the growth, inactivation and toxin production of $B$. cereus in cookedrice. Inoculated cooked-rice samples were stored at $15,25,35$ and $45{ }^{\circ} \mathrm{C}$ for the growth and toxin production of $B$. cereus, while inoculated samples (with/without 2-day storage at $35{ }^{\circ} \mathrm{C}$ ) were treated at 80,90 and $100{ }^{\circ} \mathrm{C}$ for inactivation and toxin destruction of $B$. cereus. The results indicated that production of emetic toxin was faster than enterotoxin production (no detection below $15^{\circ} \mathrm{C}$ ) at all the storage temperature $\left(15-45^{\circ} \mathrm{C}\right)$ within $72 \mathrm{~h}$. On the contrary, emetic toxin were unable to be destroyed by heat treatment $\left(80-90^{\circ} \mathrm{C}\right)$, while enterotoxin was easily to be removed. In addition, $B$. cereus was more difficult to be inactivated by heat treatment after storage since they have already made thermo stable spores. This study can provide valuable and reliable information for preventing the food poisoning illnesses and establishment of risk assessment associated with B. cereus.
\end{abstract}

Key words: Growth, inactivation, toxin production, Bacillus cereus, cooked rice. 